\title{
Nonlinear Dynamics and Bifurcation Behavior of a 2-DOF Spring Resonator with End Stopper for Energy Harvesting
}

\author{
A. El Aroudi ${ }^{1, \mathrm{a}}$, E. Blokhina ${ }^{2}$, D. O'Connell ${ }^{2}$, B. $\mathrm{Fu}^{2}$, R. Frizzell ${ }^{3}$, O. Feely ${ }^{2}$, and E. Alarcon ${ }^{4}$ \\ 1 Universitat Rovira i Virgili, Spain \\ 2 University College Dublin, Ireland \\ 3 Alcatel-Lucent, Bell Labs, Dublin, Ireland \\ 4 Universitat Politècnica de Catalunya, Spain
}

\begin{abstract}
In this paper, the model of a two-degree-of-freedom (2-DOF) spring resonator with end stopper for an energy harvesting application is presented. Then we characterize its nonlinear dynamical behavior by numerical simulations when some suitable parameters are varied. The system is formed by two resonators subject to external vibrational excitation and with an end stopper. We present the continuous time dynamical model of the system in the form of a switched fourth order differential equation. Harmonic vibrations are considered as the main ambient energy source for the system and its frequency response representing the RMS value of the displacement is first computed. The dynamical behavior is unveiled by computing state-space trajectories, timedomain series and FFT spectra and frequency response as the excitation amplitude is varied.
\end{abstract}

\section{Introduction}

Vibration-based energy harvesting is a process in which mechanical energy is transformed into electricity. This process allows the conversion of the kinetic energy from a moving body due to ambient vibrations into electrical energy through a certain electromechanical mechanism. There are different types of vibration energy harvesters. The most widely used are those based on electromagnetic, piezoelectric or electrostatic transducers. However, they all have the same key element, a mechanical resonator that effectively exploits the ambient vibrations. This topic is considered one of the key points in the development of autonomous sensors with extended lifetime [1] and the ralted growing area of research is driven by the capability of harvesters to act as an independent power supply for wireless microsystems and sensor networks, or, alternatively, to prolong the life time of batteries used in such systems.

In most of the reported studies on this topic, the energy harvesters are designed as linear single degree of freedom (SDOF) resonators by matching the resonant frequency of the harvester with that of the external excitation to extract maximum power. This maximum power extraction depends on the quality factor ( $\mathrm{Q}$ factor) of the linear resonator. In [2], [3] the authors improved piezoelectric vibration energy harvester efficiency by introducing additional masses, which make use of multiple resonant frequencies. Recently, multi-degree of freedom (MDOF) design has been also demonstrated to have the potential advantage of displacement amplification and therefore enhancing the power efficiency over a broader frequency spectrum as shown in [4] and [5]. Numerical examples are presented to illustrate that with proper selection of the design parameters of the 2-DOF resonator, the harvested power can be amplified by a factor of 20 as compared to the

a e-mail: abdelali.elaroudi@urv.cat conventional SDOF resonator and the effective bandwidth of the harvester can be widened $25 \%$ in [6]. An electromagnetic 2-DOF vibration energy harvester (VEH) was reported in [7] achieving a greater efficiency both below and above the main natural frequency compared to the SDOF VEHs.

In this work we investigate the nonlinear dynamical behavior and bifurcation phenomena of a 2-DOF mechanical resonator with an end-stopper for ambient energy harvesting applications. The system is very simple but it exhibits very complex bifurcation structures leading to subharmonic oscillations, irregular and chaotic motions. It may also have coexisting attractors within a certain range of parameter values. The rest of this paper is organized as follows. Section 2 deals with the system description and provides the mathematical model of the system in the form of a set of switched differential equations. A dimensionless formulation of the problem is presented in Section 3 where a dimensionless switched model and its corresponding boundary conditions are given. The frequency response of the system is presented in Section 4. Bifurcation phenomena are reported in Section 5. Finally concluding remarks are provided in the last section.

\section{System description}

Fig. 1 shows the schematic diagram of the 2-DOF mechanical resonator considered in this study. Two masses $m_{1}$ and $m_{2}$ are connected vertically in series to a frame through two springs with stiffness $k_{1}$ and $k_{2} . x_{1}, x_{2}$ and $x_{0}$ are the absolute displacement of the base, mass 1 and mass 2 respectively. $\ell_{1}$ and $\ell_{2}$ are the length of the two springs plus the thickness of the two masses respectively. $d$ is the gap between mass 2 and the end-stopper. The connection between these 2 masses is not solid, and this means that the spring with stiffness $k_{2}$ cannot be stretched. In Fig. 1, it can 


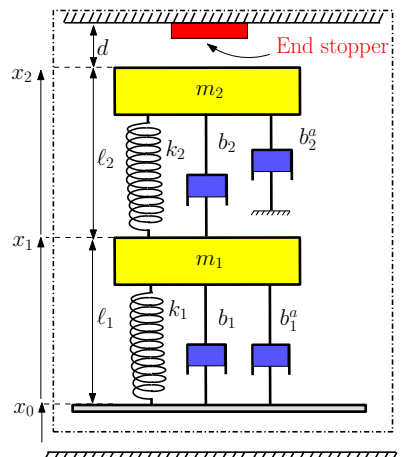

(a)

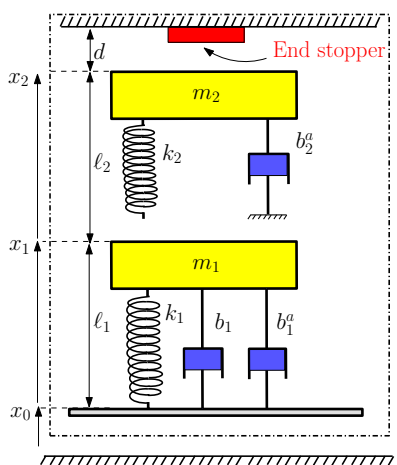

(b)
Fig. 1. Schematic diagram the two DOF spring-mass-damper system. (a) attached mass vibration, (b) detached mass vibration.

be observed that the two masses are attached and detached to and from each other through $k_{2}$. Mechanical damping in Fig. 1 consists of two parts: spring damping and air damping. In this case, a viscous damping factor is used to reproduce both types of damping: $b_{1}$ and $b_{2}$ are of spring damping, $b_{1}^{a}$ and $b_{2}^{a}$ are of air damping.

\subsection{Dynamical model}

Assuming that the air damping force is proportional to the relative velocity of the mass and taking the gravity into account, the governing equation of the system working on attached-mode as illustrated in Fig. 1 can be written as follows

$$
\begin{aligned}
\ddot{x}_{1} & =-\frac{k_{1}}{m_{1}}\left(x_{1}-x_{0}-\ell_{1}\right)-\frac{b_{1}}{m_{1}}\left(\dot{x}_{1}-\dot{x}_{0}\right)+\frac{k_{2}}{m_{1}}\left(x_{2}-x_{1}-\ell_{2}\right) \\
& -\frac{b_{2}}{m_{1}}\left(\dot{x}_{1}-\dot{x}_{2}\right)-\frac{b_{1}^{a}}{m_{1}}\left(\dot{x}_{1}-\dot{x}_{0}\right)-g \\
\ddot{x}_{2} & =-\frac{k_{2}}{m_{2}}\left(x_{2}-x_{1}-\ell_{2}\right)-\frac{b_{2}}{m_{2}}\left(\dot{x}_{2}-\dot{x}_{1}\right)-\frac{b_{2}^{a}}{m_{2}}\left(\dot{x}_{2}-\dot{x}_{0}\right)-g
\end{aligned}
$$

Let us define the following new state variables

$$
y_{1}=x_{1}-x_{0}-\ell_{1}, \quad y_{2}=x_{2}-x_{0}-\ell_{2}-\ell_{1}
$$

By substituting these new variables in (1)-(2), the following differential equations are obtained for the motion of the resonator

$$
\begin{aligned}
\left(\ddot{y}_{1}+\ddot{y}_{0}\right) & =-\frac{k_{1}}{m_{1}} y_{1}-b_{1} \dot{y}_{1}+\frac{k_{2}}{m_{1}}\left(y_{2}-y_{1}\right)-\frac{b_{2}}{m_{2}}\left(\dot{y}_{1}-\dot{y}_{2}\right) \\
& -\frac{b_{1}^{a}}{m_{1}} \dot{y}_{1}-g \\
\left(\ddot{y}_{2}+\ddot{y}_{0}\right) & =-\frac{k_{2}}{m_{2}}\left(y_{2}-y_{1}\right)-\frac{b_{2}}{m_{2}}\left(\dot{y}_{2}-\dot{y}_{1}\right)-\frac{b_{2}^{a}}{m_{2}} \dot{y}_{2}-g
\end{aligned}
$$

where $\ddot{y}_{0}$ is the external acceleration which can be represented by $\ddot{y}_{0}=A_{\text {ext }} \cos \left(\omega_{\text {ext }} t+\phi_{0}\right)$. If it is considered that the air damping force is proportional to the absolute velocity of mass, then one has for mass 1

$$
F_{\text {air }}=-b_{1}^{a} \dot{y}_{1}-b_{1}^{a}\left(\dot{y}_{1}-\dot{y}_{0}\right)-b_{1}^{a} \dot{y}_{0}=b_{1}^{a} \dot{y}_{1}-b_{1}^{a} \dot{y}_{0}
$$

When the mass $m_{2}$ is in contact with with the end stopper, the stopper force $F_{\text {im }}$ can be written as follows

$$
F_{\text {im }}=k_{\text {im }}\left(y_{2}-y_{\max }\right)+b_{\text {im }} \dot{y}_{2}
$$

where $k_{\text {im }}$ is the stiffness and $b_{\text {im }}$ is the damping factor of the stopper. Finally the system governing equations for the attached vibration can be written as follows

$$
\begin{array}{r}
\ddot{y}_{1}+\frac{b_{1}+b_{1}^{a}}{m_{1}} \dot{y}_{1}+\frac{k_{1}}{m_{1}} y_{1}+\frac{k_{2}}{m_{1}}\left(y_{1}-y_{2}\right)+\frac{b_{2}}{m_{1}}\left(\dot{y}_{1}-\dot{y}_{2}\right)= \\
-A_{\mathrm{ext}} \cos \left(\omega_{\mathrm{ext}} t+\phi_{0}\right)-g \\
\ddot{y}_{2}+\frac{b_{2}^{a}}{m_{2}} \dot{y}_{2}+\frac{k_{2}}{m_{2}}\left(y_{2}-y_{1}\right)+\frac{b_{2}}{m_{2}}\left(\dot{y}_{2}-\dot{y}_{1}\right)+\frac{F_{\mathrm{im}}}{m_{2}}= \\
-A_{\mathrm{ext}} \cos \left(\omega_{\mathrm{ext}} t+\phi_{0}\right)-g
\end{array}
$$

and for the detached vibration mode one has

$$
\begin{aligned}
\ddot{y}_{1}+\frac{b_{1}+b_{1}^{a}}{m_{1}} \dot{y}_{1}+\frac{k_{1}}{m_{1}} y_{1} & =-A_{\mathrm{ext}} \cos \left(\omega_{\mathrm{ext}} t+\phi_{0}\right)-g(10) \\
\ddot{y}_{2}+\frac{b_{2}^{a}}{m_{2}} \dot{y}_{2}+\frac{F_{\mathrm{im}}}{m_{2}} & =-A_{\mathrm{ext}} \cos \left(\omega_{\mathrm{ext}} t+\phi_{0}\right)-g(11)
\end{aligned}
$$

\subsection{Boundary conditions}

According to the system description illustrated in Fig. 1, the two masses are attached to each other under the condition

$$
x_{2}-x_{1}<\ell_{2}
$$

and mass 2 is not in contact with the end stopper under the condition

$$
x_{2}-x_{0}<\ell_{1}+\ell_{2}+d
$$

In terms of the new variables $y_{1}$ and $y_{2}$, the previous boundary conditions become

$$
\begin{array}{r}
y_{2}-y_{1}<\ell_{2} \\
y_{2}<d
\end{array}
$$

and the stopper force $F_{\text {im }}$ is given by

$$
F_{\text {im }}= \begin{cases}0 & \text { if } y_{2}-d<0 \\ k_{\mathrm{im}}\left(y_{2}-d\right)+b_{\text {im }} \dot{x}_{2} & \text { if } y_{2}-d>0 .\end{cases}
$$

The previous boundary conditions define in the state space the so called switching surfaces which when crossed by a system trajectory, its dynamics change non-smoothly from one form of linear behavior to another. The state space is divided into different subspaces by these switching surfaces. Although the system is linear in each subspace, the global behavior is highly nonlinear. The system can be integrated in closed form within each subspace and only the switching instants have to be computed using numerical methods such as the Newton-Raphson algorithm. 


\section{Dimensionless Formulation}

The previous equations of motion include 12 physical parameters. In order to reduce this number, let us define the following dimensionless parameters and variables

$$
\begin{array}{r}
\omega_{01}=\sqrt{\frac{k_{1}}{m_{1}}}, \quad \omega_{02}=\sqrt{\frac{k_{2}}{m_{2}}}, \quad \Omega_{\mathrm{ext}}=\frac{\omega_{\mathrm{ext}}}{\omega_{01}}=1+\sigma, \\
\Omega_{21}=\frac{\omega_{02}}{\omega_{01}}, \quad \alpha=\frac{A_{\mathrm{ext}}}{d \omega_{01}^{2}}, \quad \gamma=\frac{g}{d \omega_{01}^{2}}, \quad \kappa_{1}=\frac{k_{2}}{k_{1}}, \\
\beta_{1}=\frac{b_{1}+b_{1}^{\prime}}{2 \sqrt{k_{1} m_{1}}}, \quad \beta_{11}=\frac{b_{2}}{2 \sqrt{k_{1} m_{1}}}, \beta_{2}=\frac{b_{2}^{\prime}}{2 m_{2} \omega_{01}}, \\
\beta_{22}=\frac{b_{2}}{m_{2} \omega_{01}}, \quad z_{1}=-\frac{y_{1}}{d}, \quad z_{2}=-\frac{y_{2}}{d \omega_{0}}, \\
\dot{z}_{1}=\frac{\dot{y}_{1}}{d \omega_{0}}, \quad \dot{z}_{2}=\frac{\dot{y}_{2}}{d \omega_{0}}, \quad t^{\prime}=\omega_{0} t .
\end{array}
$$

where $z_{1}$ and $z_{2}$ are the dimensionless displacements. Let us also consider $\phi_{0}=0$ for simplicity. Therefore the dynamical model of the system can be written as follows. In the attached mode $z_{2}<z_{1}$

$$
\begin{array}{r}
\ddot{z}_{1}+2 \beta_{1} \dot{z}_{1}+z_{1}+b_{1} \dot{z}_{1}+\kappa\left(z_{1}-z_{2}\right)+\beta_{11}\left(\dot{z}_{1}-\dot{z}_{2}\right)= \\
-\alpha \cos ((1+\sigma) t)-\gamma \\
\ddot{z}_{2}+2 \beta_{2} \dot{z}_{2}+\Omega_{21}^{2}\left(z_{2}-z_{1}\right)+\beta_{22}\left(\dot{z}_{2}-\dot{z}_{1}\right)+\tilde{F}_{\mathrm{im}}= \\
-\alpha \cos ((1+\sigma) t)-\gamma
\end{array}
$$

and for the detached-vibration mode $z_{2}>z_{1}$ one has

$$
\begin{aligned}
\ddot{z}_{1}+2 \beta_{1} \dot{z}_{1}+z_{1} & =-\alpha \cos ((1+\sigma) t)-\gamma \\
\ddot{z}_{2}+2 \beta_{2} \dot{z}_{2}+\tilde{F}_{\mathrm{im}} & =-\alpha \cos ((1+\sigma) t)-\gamma
\end{aligned}
$$

The derivation (overdot) is now taken with respect to the normalized time $t^{\prime}$. For convenience of notation, in the following we drop the use of prime in the definition of dimensionless time. Considering the normalization of $z_{2}$, the stopper force taking place when $z_{2}>1$ can be written as follows

$$
\tilde{F}_{\text {im }}=\kappa_{\text {im }}\left(z_{2}-1\right)+\beta_{\text {im }} \dot{z}_{2}
$$

where $\kappa_{\mathrm{im}}$ is the normalized stiffness and $\beta_{\mathrm{im}}$ is the normalized damping factor of the stopper given respectively by

$$
\kappa_{\mathrm{im}}=\frac{k_{i m} m_{1}}{m_{2} k_{1}} \quad \text { and } \quad \beta_{\mathrm{im}}=\frac{b_{\text {im }}}{m_{2} \sqrt{k_{1} / m_{1}}}
$$

The switching surfaces in the normalized space where the switching from one linear configuration to another takes place are defined by

$$
\begin{gathered}
\Sigma_{1}=\left\{\mathbf{z} \mid z_{1}=z_{2}\right\} \\
\Sigma_{2}=\left\{\mathbf{z} \mid z_{2}=1\right\}
\end{gathered}
$$

where $\mathbf{z}=\left(z_{1}, \dot{z}_{1}, z_{2}, \dot{z}_{2}\right) \in \mathbb{R}^{4}$ is the dimensionless vector of the state variables. Intersecting one of these two surfaces, the system dynamics change non-smoothly from one linear configuration to another in the normalized statespace.

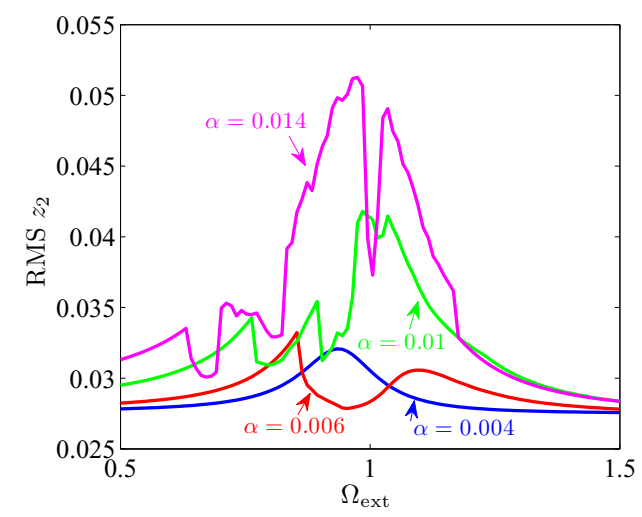

Fig. 2. Frequency response of the system representing the RMS value of the dimensionless displacement $z_{2}$ for $\alpha=0.004,0.006$, 0.01 , and 0.014 .

\section{The frequency response}

The dimensionless parameter values of the 2-DOF coupled resonator considered in this study are listed in Table 1. Long-time integration is used to obtain the frequency response of the system. The system is simulated during a total of 150 cycles. The first 100 cycles are considered as transient regime and the last 50 cycles are used as steadystate data to compute the RMS values of the state variables then those corresponding to the displacement $z_{2}$ are plotted afterward. This procedure is repeated for different values of the exciting force amplitude and the result is depicted in Fig. 2. For low values of the excitation force amplitude ( $\alpha=0.005$ in this case), the frequency response is a typical response of a linear system. This is because in this case the boundaries are not crossed and the system works in one of the linear subspace without any switching. As the excitation force amplitude $\alpha$ is increased, the boundary $\Sigma_{1}$ is crossed and the system switches between different configurations giving rise to nonlinear behavior. The frequency responses shown in Fig. 2 for $\alpha=0.006,0.01$ and 0.014 correspond to the nonlinear case. It can be observed that the curve of the frequency response is characterized by a double peak value.

\section{Exploring the system dynamics}

In order to explore the different dynamical behavior that the system can exhibit, a bifurcation diagram has been obtained by taking $\alpha$ as a bifurcation parameter which was varied within a wide range from 0.004 to 0.014 . The result is depicted in Fig. 3. This bifurcation diagram is obtained by sampling the state trajectories stroboscopically at the driving frequency. Long transient data are eliminated and only 50 samples are used as steady state. Time-domain waveforms, state-space trajectories and FFT spectra of the state variables are also used to explain the observed dynamics. The results are shown in Fig. 4-Fig. 10 for different values of the exciting amplitude $\alpha$. For low values of the excitation force amplitude, the system steady-state behavior is a $T$-periodic orbit in the linear regime. As mentioned before, this is because in this case the switching surfaces defined in (22)-(23) are not reached and the system 
Table 1. The used parameter values.

\begin{tabular}{|l|l|l|l|l|l|l|l|l|l|l|l|}
\hline$\Omega_{21}$ & $\Omega_{02}$ & $\beta_{1}$ & $\beta_{11}$ & $\beta_{2}$ & $\beta_{22}$ & $\gamma$ & $\kappa$ & $\beta_{\text {im }}$ & $\kappa_{\text {im }}$ & $\sigma$ & $\alpha$ \\
\hline 2.236 & 2.236 & 0.1 & 0.007 & 0.007 & 0.07 & 0.025 & 0.5 & 0.078 & 283 & 0.0 & 0.002 \\
\hline
\end{tabular}

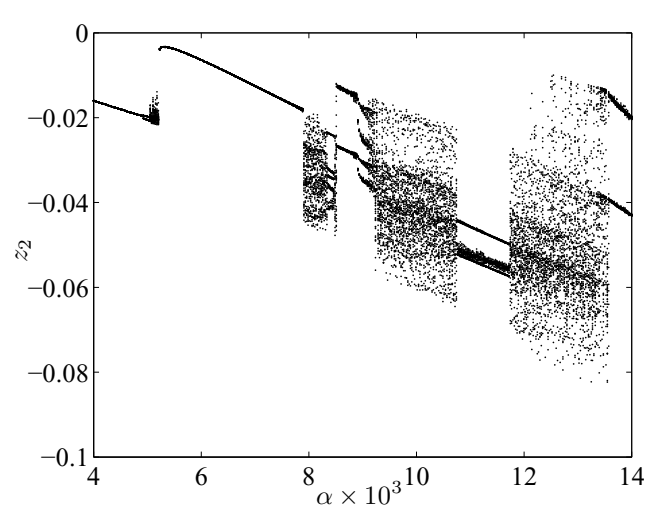

(a)

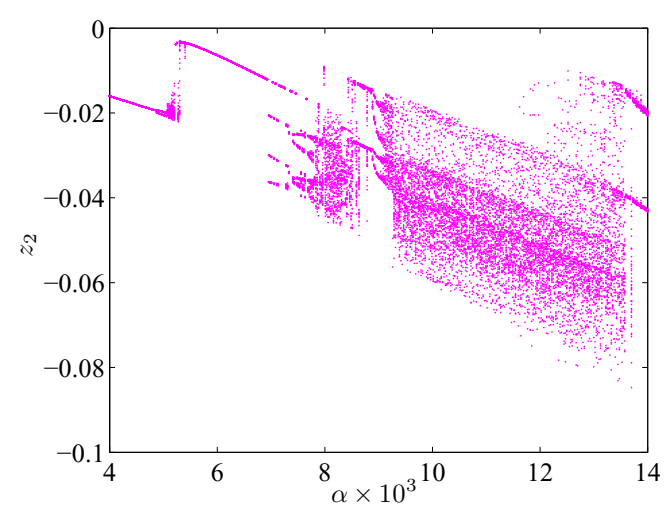

(b)

Fig. 3. Bifurcation diagram taking $\alpha$ as a bifurcation parameter and representing the dimensionless displacement of the second resonator $z_{2}$. To detect possible coexisting attractors, in the first diagram the initial conditions are updated by the final values corresponding to the last parameter value, while in the second diagram the initial conditions of the state variables are set zero for each value of the bifurcation parameter.

works without switching between the different configurations. This can be observed in the time domain waveforms of Fig. 4 where the state variable $z_{2}$ does not intersect the state variable $z_{1}$ neither it does with the critical value 1 .

As the parameter $\alpha$ is increased, the boundary defined by the first switching surface $\Sigma_{1}$ defined by $z_{1}=z_{2}$ is crossed at a critical value $\alpha \approx 0.00472$ and the system switches between the two different configurations defined by (8)-(9) and (10)-(11). At values of $\alpha$ slightly larger than this critical value, the system dynamical behavior is still a stable $T$-periodic regime but the FFT spectra of the state variables contains high harmonic contents in contrast to the linear regime where a single tone spectrum can be observed at the driving frequency. However, when $\alpha$ is further increased, the system $T$ - periodic orbit loses its stability through a period doubling bifurcation leading to subharmonic oscillation occurrence manifested by a $2 T$-periodic orbit as shown in Fig. 5. The FFT spectrum shows in this
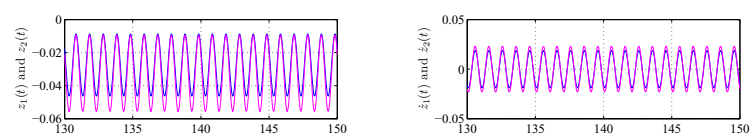

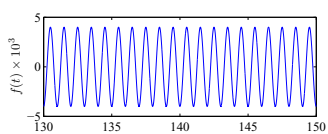

(a)

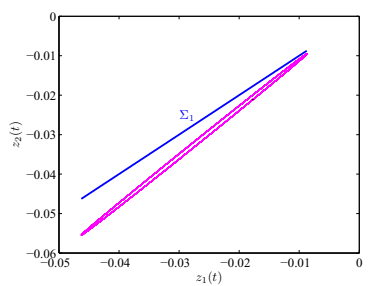

(c)

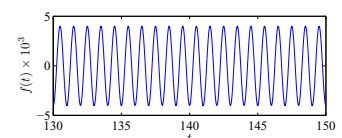

(b)

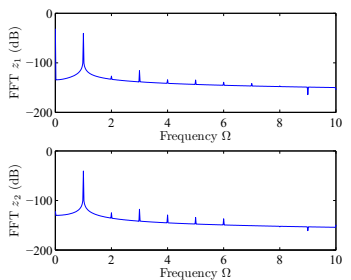

(d)
Fig. 4. Steady-state behavior of the system for $\alpha=0.004$.
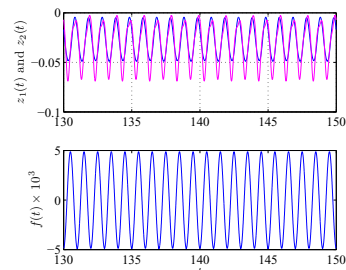

(a)

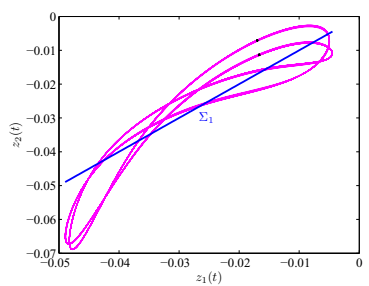

(c)
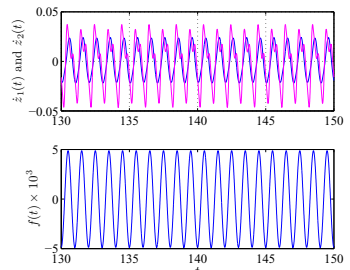

(b)

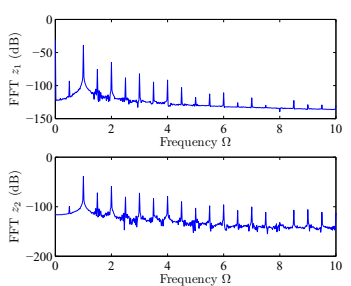

(d)
Fig. 5. Steady-state behavior of the system for $\alpha=0.00487$.

case a harmonic content at one half the driving frequency and its multiple integer values.

When the bifurcation parameter is further increased beyond $\alpha \approx 0.00486$, the $2 T$-periodic orbit losses its stability giving rise to sudden occurrence of chaotic regimes and the spectrum is spread within a wide frequency range covering from zero to a certain bandwidth as it is depicted in Fig. 6.

If $\alpha$ is increased, the system becomes $T$-periodic with a remarkably large amplitude oscillation in the second resonator displacement $z_{2}$ (Fig.7).

In some parameter range this orbit coexists with a larger amplitude $4 T$-periodic orbit as it is illustrated in Fig. 8 for $\alpha=0.0074$. It can be observed that in all the previous 

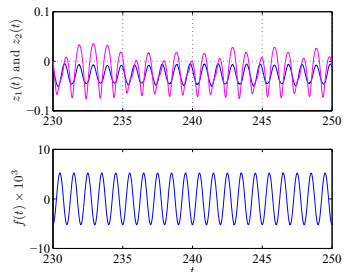

(a)

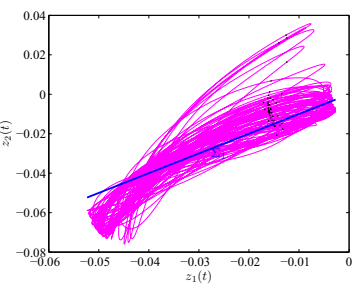

(c)
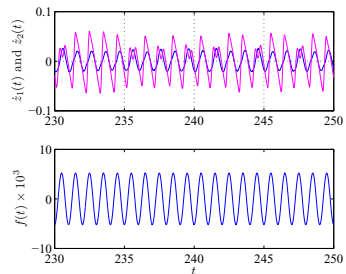

(b)

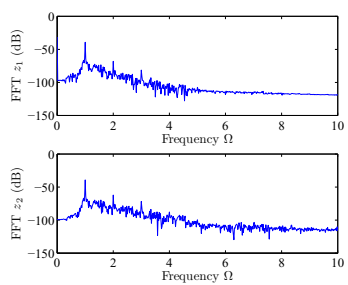

(d)
Fig. 6. Steady-state behavior of the system for $\alpha=0.0052$.
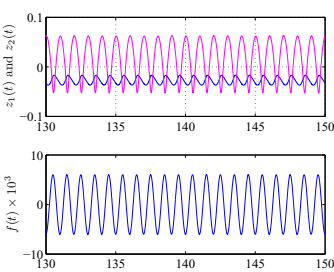

(a)

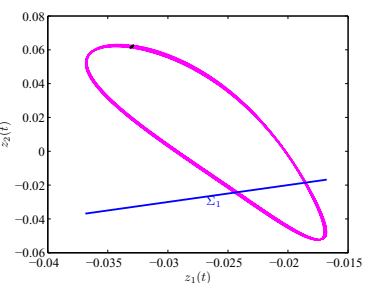

(c)
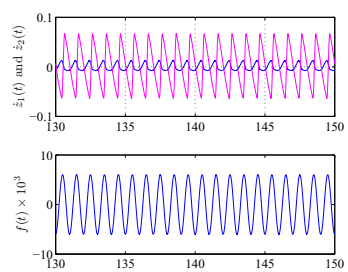

(b)

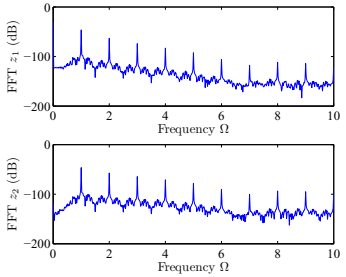

(d)
Fig. 7. Steady-state behavior of the system for $\alpha=0.006$.

cases, only the boundary defined by the switching surface $\Sigma_{1}$ is crossed while the end stopper boundary condition is not reached.

The $T$ - periodic orbit in this region of the parameter space can loose its stability giving rise to a chaotic regime shown in Fig. 9 for $\alpha=0.012$. At this value of the bifurcation parameter, the switching surface $\Sigma_{2}$ is also crossed and an expansion of the chaotic attractor can be observed. By further increasing $\alpha$, a stable a large amplitude $2 T$-periodic orbit emerges. This orbit hits both surfaces $\Sigma_{1}$ and $\Sigma_{2}$ as it is shown in Fig. 10.

\section{Conclusions}

In this work we have studied a periodically driven 2-DOF spring resonator with end stopper for an energy harvesting application from a nonlinear dynamics perspective. We have shown that the system can present a rich variety of nonlinear behavior such as bifurcation phenomena and chaotic

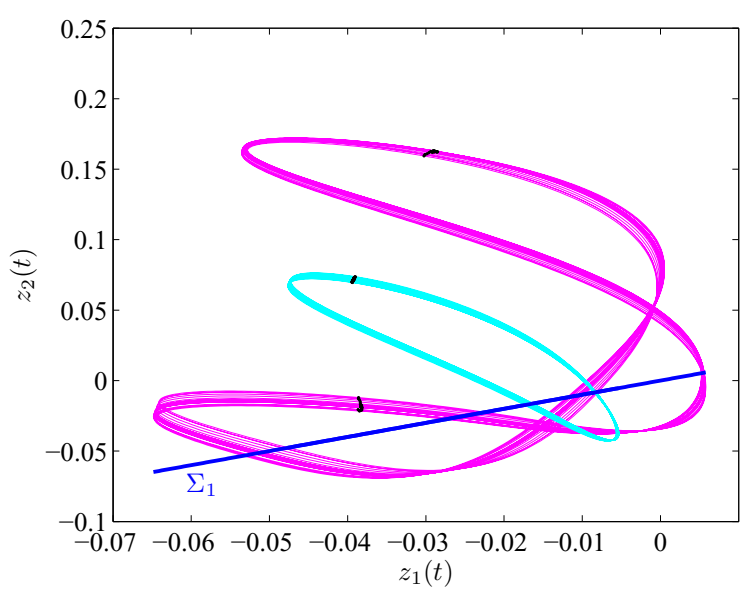

Fig. 8. Coexisting attractors for $\alpha=0.0074$.
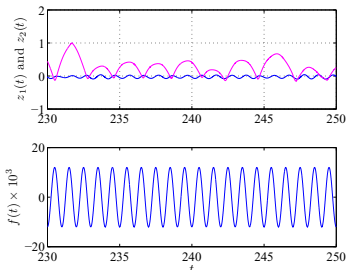

(a)

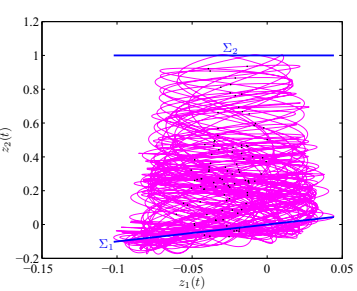

(c)
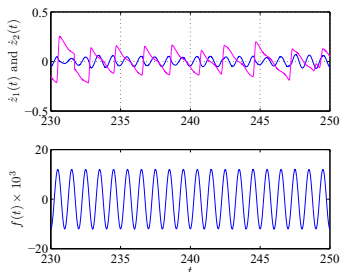

(b)

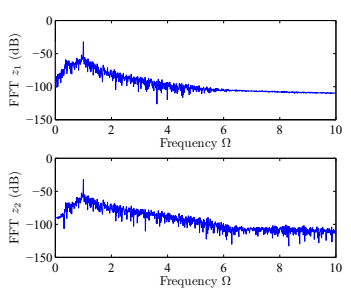

(d)
Fig. 9. Steady-state behavior of the system for $\alpha=0.012$.
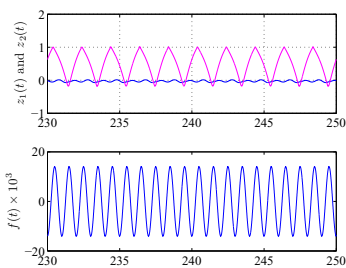

(a)

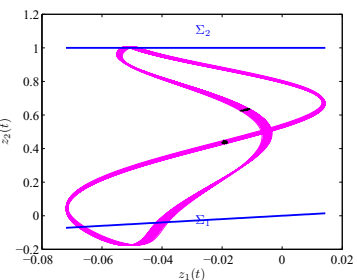

(c)
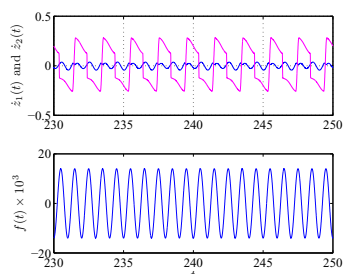

(b)

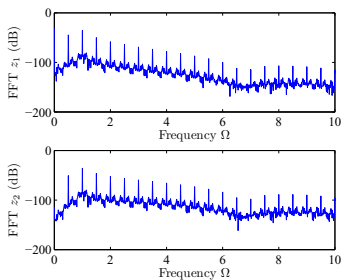

(d)
Fig. 10. Steady-state behavior of the system for $\alpha=0.014$. 
behavior. Numerical simulations have been carried out using a nonlinear switched model to unveil the different dynamical behavior of the system. Time-domain waveforms, FFT spectra, and bifurcation diagrams have been obtained. Despite the system simplicity, it presents complex phenomena and nonsmooth transitions to chaotic regimes. Local and global stability analysis of periodic orbits is a subject of further study.

\section{Acknowledgments}

This work was partially supported by the Ministerio de Ciencia e Innovación under grant CSD2009-00046 and by Science Foundation Ireland.

\section{References}

1. P. D. Mitcheson, E. M. Yeatman, G. K. Rao, A. S. Holmes and T. C. Green, "Energy Harvesting From Human and Machine Motion for Wireless Electronic Devices", Proceedings of the IEEE, 96(9), 1457-1586, 2008.

2. P. S. Ma, E. J. Kim, and Y. Y. Kim, "Power-amplifying strategy in vibration-powered energy harvesters," in Active and Passive Smart Structures and Integrated Systems, 2010.

3. Z. Wong, J. Yan, K. Soga, and A. Seshia, "A multi-degree-of-freedom electrostatic MEMS power harvester," Proc. PowerMEMS 2009,(1) , pp. 300-303, 2009.

4. L. Tang and Y. Yang, "A multiple-degree-of-freedom piezoelectric energy harvesting model" , Journal of Intelligent Material Systems and Structures, 23(14), pp. 1631-1647, 2012.

5. C. Acar and A. Shkel, "Inherently robust micromachined gyroscopes with 2-DOF sense-mode oscillator," Microelectromechanical Syst. J., 15(2), pp. 380-387, 2006.

6. A. Khaligh, P. Zeng, and C. Zheng, "Kinetic energy harvesting using piezoelectric and electromagnetic technologies-state of the art," Ind. Electron. IEEE , 57(3), pp. 850- 860, 2010.

7. A. Schofield, A. Trusov, and A. Shkel, "Effects of operational frequency scaling in multi-degree of freedom MEMS gyroscopes," IEEE Sensors Journal, 8(10), pp. 1672-1680, 2008. 